\title{
Explaining the Cardiovascular Risk Associated with Rheumatoid Arthritis: Traditional Risk Factors Versus Markers of Rheumatoid Arthritis Severity
}

\author{
Daniel H. Solomon, Joel Kremer, Jeffrey R Curtis, Marc C. Hochberg, George Reed, Peter \\ Tsao, Michael E. Farkouh, Soko Setoguchi, and Jeffrey D. Greenberg \\ Division of Rheumatology, Immunology, and Allergy, Brigham and Women's Hospital (DHS and \\ PT); Division of Pharmacoepidemiology, Brigham and Women's Hospital (DHS, SS); Division of \\ Rheumatology, Albany Medical College (JK); Division of Clinical Immunology and Rheumatology, \\ University of Alabama at Birmingham (JRC); Division of Rheumatology and Clinical Immunology, \\ Department of Medicine and Division of Gerontology, Department of Epidemiology and Preventive \\ Medicine, University of Maryland School of Medicine $(\mathrm{MCH})$; Clinical Trials Unit, Mount Sinai Heart \\ (MEF); Division of Rheumatology, New York University School of Medicine (JDG)
}

\section{Abstract \\ Background-Cardiovascular (CV) disease has a major impact on patients with rheumatoid arthritis (RA), however, the relative contributions of traditional CV risk factors and markers of RA severity are unclear. We examined the relative importance of traditional CV risk factors and RA markers in predicting CV events.}

\begin{abstract}
Methods-A prospective longitudinal cohort study was conducted in the setting of the CORRONA registry in the United States. Baseline data from subjects with RA enrolled in the CORRONA registry were examined to determine predictors of $\mathrm{CV}$ outcomes, including myocardial infarction (MI), stroke or transient ischemic attack (TIA). Possible predictors were of two types: traditional CV risk factors and markers of RA severity. The discriminatory value of these variables was assessed by calculating the area under the receiver operating characteristic curve (c-statistic) in logistic regression. We then assessed the incidence rate for $\mathrm{CV}$ events among subjects with an increasing number of traditional $\mathrm{CV}$ risk factors and/or RA severity markers.
\end{abstract}

Results-The cohort consisted of 10,156 patients with RA followed for a median of 22 months. We observed 76 primary CV events during follow-up for a composite event rate of 3.98 (95\% CI $3.08-4.88)$ per 1,000 patient-years. The c-statistic improved from 0.57 for models with only CV risk factors to 0.67 for models with $\mathrm{CV}$ risk factors plus age and gender. The c-statistic improved further to 0.71 when markers of RA severity were also added. The incidence rate for CV events was 0 (95\% CI $0-5.98)$ for persons without any CV risk factors or markers of RA severity, while in the group with two or more $\mathrm{CV}$ risk factors and 3 or more markers of RA severity the incidence was 7.47 (95\% CI 4.21-10.73) per 1,000 person-years.

Conclusions-Traditional CV risk factors and markers of RA severity both contribute to models predicting $\mathrm{CV}$ events. Increasing numbers of both types of factors are associated with greater risk.

Correspondence: Daniel H. Solomon, MD, MPH, Division of Rheumatology, Brigham and Women's Hospital, 75 Francis Street, Boston MA 02115; T: 617-732-5356; F: 617-732-5505; dhsolomon@ partners.org.

The Corresponding Author has the right to grant on behalf of all authors and does grant on behalf of all authors, an exclusive licence (or non exclusive for government employees) on a worldwide basis to the BMJ Publishing Group Ltd to permit this article (if accepted) to be published in ARD and any other BMJPGL products and sublicences such use and exploit all subsidiary rights, as set out in our licence (http://ARD.bmjjournals.com/ifora/licence.pdf). 


\section{INTRODUCTION}

Cardiovascular (CV) disease represents a major source of morbidity and mortality for patients with RA.1-4 Myocardial infarction (MI) and stroke are common events and thus difficult for a single clinician to associate with a given disease, such as RA. However, a series of large epidemiologic studies over the last several decades strongly support an elevation in CV risk with RA.3, 4 These studies have come from both community-based and referral populations, and consistently find that RA patients have a 1.5 to 3-fold increased risk for CV events compared with non-RA controls. In addition to the increased risk of CV events in RA, silent cardiac ischemia and fatal $\mathrm{CV}$ presentations may be more common in RA than in non-RA subjects. $5^{-7}$

In spite of the clear association between $\mathrm{CV}$ events and RA, elucidating the link between these conditions has been challenging. Some, but not all, studies suggest that the heightened CV risk is mediated by an effect of RA on traditional CV risk factors, such as hyperlipidemia and insulin resistance. ${ }^{8-} 10$ Other data demonstrate that markers of RA severity associate with surrogate markers of $\mathrm{CV}$ risk; for example, elevated acute phase reactants correlate with an increased carotid intima medial thickness.11 In patients with new onset inflammatory polyarthritis, CRP levels are directly related to risk of death.12 Other results have also supported the relationship between RA disease activity and CV risk. ${ }^{13}$ There are also data suggesting an association between rheumatoid factor (RF) and CV events. ${ }^{14}$ Some medications used for RA have been linked with CV risk - glucocorticoids and nonsteroidal anti-inflammatory drugs. However, these agents do not appear to completely explain the CV risk associated with RA.13, 15, 16

Several prior studies have demonstrated an association between CV risk factors, markers of RA severity and atherosclerosis. ${ }^{17} 18$ These studies have been consistent in their findings, but none have used actual $\mathrm{CV}$ events as an outcome.

A more complete understanding of risk factors for CV events in RA will facilitate development and testing of intervention strategies. On a population level, better control of traditional CV risk factors will likely lead to fewer $\mathrm{CV}$ events in RA, but it is unclear whether a strategy of enhanced control of RA disease activity will also lead to reduced CV risk. Moreover, there is no information on the relative importance of targeting traditional $\mathrm{CV}$ risk factors versus markers of RA severity to affect $\mathrm{CV}$ risk. We thus examined the relative importance of baseline traditional CV risk factors compared with baseline markers of RA severity to determine their relative predictive value for $\mathrm{CV}$ events in a very large longitudinal cohort. This work was guided by the hypothesis that both sets of factors would add independent predictive value to models of CV events among patients with RA.

\section{METHODS}

\section{Study Cohort}

We examined data from CORRONA, the Consortium of Rheumatology Researchers of North America. Since 2002, CORRONA has enrolled over 17,000 patients with RA through 268 participating academic and community rheumatologists at 103 sites in the United States. Rheumatologists and their staff receive training on patient recruitment and form completion. They are reimbursed for the collection of data at the time of the routine clinical encounter on consecutive patients in a prospective manner. For the purposes of the current analyses, we included only patients diagnosed with RA, and not Psoriatic Arthritis, who contributed information to CORRONA on at least three rheumatology visits. All patients satisfied the ACR criteria according to the enrolling Rheumatologist. No specific selection criteria are applied to enrolling patients with RA. Follow-up began with the baseline CORRONA visit and continued through December 31, 2006, an endpoint, or loss to follow-up, whichever came first. 
All subjects have given written informed consent and the study protocol has been approved by the appropriate Institutional Review Board.

\section{Endpoints}

The $\mathrm{CV}$ events considered in this analysis include major ischemic events, but not heart failure or peripheral arterial disease. The rationale for this decision is that other predictive models of $\mathrm{CV}$ events, such as the Framingham Risk Score, were developed to predict ischemic CV events. ${ }^{19}$ In addition, while ischemia is an important factor in heart failure, the risk factors can be different. We examined follow-up rheumatologist assessment forms for reports of new CV events, including myocardial infarction (MI), stroke, or transient ischemic attack (TIA). There were specific questions for each of these endpoints on the forms for the study period (20022006), but not for unstable angina. In addition, because death certificates were not available for the majority of patients, we chose to exclude CV-related deaths for this study. These reports prompted a follow-up inquiry to the rheumatologist to confirm the report, determine if it was a new event and the date of the event. Medical records were then requested regarding the event.

To test the validity of the rheumatologist-confirmed CV events, a three-person Adjudication Committee comprised of two board-certified cardiologists and an internal medicine specialists reviewed the $56 \%$ ( 42 of 75 ) of cases of MI, stroke and TIA reported by participating rheumatologists for which hospitalization records where available. All events were classified into one of four possible categories: definite, probable, possible and unlikely $\mathrm{CV}$ events. Events adjudicated as definite $(31 \%)$ or probable $(65 \%)$ events were considered as true events for the purposes of validation (see Supplemental File for further explanation of the adjudication criteria). Case definition criteria for MI, stroke, and TIA were applied by the adjudication committee as previously defined. The MI adjudication criteria were consistent with definitions from the American Heart Association guidelines for both ST segment elevation and non-STsegment elevation MI. The stroke case definition was consistent with the American Heart Association/American Stroke Association guidelines. ${ }^{20}$ (See Supplemental File for details of adjudication criteria.) The positive predictive value for confirmed cases where records were available was 96\%.(See Supplemental File for details of confirmation process.)

The primary analysis used all CV events confirmed by rheumatologists. Sensitivity analyses used definite or probable events based on the adjudication committee review.

\section{Potential Predictors}

Both patients and their rheumatologists complete assessment forms at all visits, typically occurring every 3-6 months (mean 4.5 months). Age was defined at the time of enrollment. The baseline forms focus on RA disease characteristics, functional status, disease activity, and medication use, as well as family history of diseases, including CV disease. Acute phase reactants (CRP or ESR) were not available in all subjects and thus were not considered in these analyses. We combined data regarding autoantibody status -- rheumatoid factor and anti-CCP antibody -- into a composite "seropositive" variable. Seven variables were available in most patients and considered as potential markers of RA severity: longer disease duration ( $>5$ years) 3 , the presence of radiographic joint erosions, the presence of subcutaneous nodules, prior total joint replacement, modified Health Assessment Questionnaire score $\geq 2,21$ a Clinical Disease Activity Index (CDAI) score > 22,22 and seropositivity. The CDAI combines swollen and tender joint counts, a physician's assessment of global arthritis activity and a patient's assessment of arthritis activity, but does not require an inflammatory marker.

Traditional CV risk factors were also collected from the baseline rheumatologist and patient questionnaire. These included six variables -- hypertension, diabetes, treated hyperlipidemia, current tobacco use, known CV disease (such as prior MI), and a family history of premature 
$\mathrm{CV}$ events (at age less than 50 years). The hyperlipidemia variable was based on the use of a lipid-lowering agent. No information was available on subject's actual blood pressure or lipid levels. Known CV disease was based on a report of prior MI, stroke, TIA, or coronary intervention, such as stenting or a coronary artery bypass graft.

\section{Statistical Analysis}

The patient cohort was described according to their RA and CV characteristics. Since our goal was to examine the predictive accuracy of groups of variables measured at baseline, i.e., traditional CV risk factors and markers of RA severity, and not a given variable, we did not use the univariate parameter estimates to determine which variables to place in the multivariable models. The potential markers of RA severity were chosen a priori and based on variables without substantial missing data. This tends to lead to a less overfit model.

We assessed the accuracy of these variables using several different model fit statistics, evaluating both the discrimination and calibration of the various models. Discrimination refers to the ability of a model to accurately predict which subjects experience an endpoint. The area under the receiver operating characteristic curve, measured as the c-statistic, is a commonly used measure of model discrimination that can be obtained from the logistic model. ${ }^{23}$ The cstatistic is considered a measure of how well a model with one or several variables can discriminate between one outcome and another, with a c-statistic of 0.50 being no better than chance and 1.0 being perfect. Calibration describes a model's ability to determine levels of risk. The Akaike Information Criterion (AIC), a goodness of fit test, was assessed from the logistic regression models. ${ }^{24}$ The AIC allows one to compare a given model's predicted values with actual values.

Finally, the incidence rate of CV events and 95\% confidence intervals (CI) were calculated for subjects with different numbers of each type of risk factor. For this analysis, age $\geq 75$ years and male gender were considered traditional $\mathrm{CV}$ risk factors. Thus, there were eight potential traditional $\mathrm{CV}$ risk factors and seven markers of RA severity. By examining the incidence rate in this stratified analysis, we are able to adjust for these confounders without making any assumptions about the covariate matrix. All analyses were run using SAS Statistical Software (version 9.0, Cary NC).

\section{RESULTS}

The study cohort included 10,156 subjects followed for a median of 22 months. At cohort entry, the subjects had a mean age of 59 years and $75 \%$ were female. The median disease duration at the start of follow-up was 84 months and $72 \%$ were seropositive. The median modified HAQ was 0.8 with rheumatoid nodules noted among $20.1 \%$ and total joint replacement among $30.4 \%$. Traditional CV risk factors are also listed in Table 1. Diabetes was present in $7.1 \%$ of subjects, hypertension in $29.8 \%$, hyperlipidemia in $9.2 \%$, and current tobacco use in $16.1 \%$. Family history of premature CV events was noted by $5.1 \%$ of subjects. As well, $8.5 \%$ of subjects reported prior CAD as evidenced by an MI, stroke, TIA, angina, or coronary intervention. Baseline use of treatments for RA included: $63 \%$ methotrexate, $35 \%$ TNF blocker, and $17 \%$ other DMARDs.

During the 18,829 patient-years of follow-up, 29 MIs and 47 strokes or TIAs were reported and confirmed by rheumatologists. As shown in Table 2, the event rates was 3.98 (95\% CI $3.08-4.88)$ per 1,000 person-years. The event rates were higher for patients 75 years and over versus those younger than 75 years and for men versus women (see Table 2).

Several logistic models were fit -- traditional CV related factors only (Model A), markers of RA severity (Model B), both types of variables (Model C), both types of variables plus age 
and gender (Model D). The goal of these models was not to determine the risk associated with an individual factor, but rather to compare the models' characteristics. The area under the receiver operating characteristic curves improved with each subsequent model: Model A cstatistics $=0.57$, Model B c-statistic $=0.64$, Model C c-statistic $=0.66$, and Model D c-statistic $=0.71$ (see Figure). In an alternative analysis, the c-statistic improved from 0.67 to 0.71 ( $p=$ 0.0061 ) when markers of RA severity were added to the traditional CV risk factors plus age and gender. The increase in c-statistic was larger when RA severity measures were added to age and gender (change in c-statistic $=0.07$ ) than when the $\mathrm{CV}$ risk factors were added (change in c-statistic $=0.04$ ). As well, the AIC improves (lower values) incrementally across the models types of variables are added: Model A AIC $=898$, Model B AIC $=895$, Model C AIC $=897$, Model D AIC 887. (Each model's AIC and c-statistic were calculated using the same cohort to avoid sample size impacting AIC.) The sensitivity analysis using only definite or probably $\mathrm{CV}$ events according to the adjudication committee yielded similar results (see Supplemental File).

Finally, we calculated the number of traditional CV risk factors and RA markers (see Table 3) and then the incidence rates for $\mathrm{CV}$ events based on the number of factors (see Table 4). While the increase in risk was not continuous across all categories of risk factors because of few events, there was a clear trend toward an increased risk with more traditional CV risk factors and markers of RA severity. Subjects without any risk factors or markers of RA severity had an incidence rate of 0 (95\% CI 0-5.98) CV events per 1,000 person-years, while subjects with 2 or more traditional CV risk factors and 3 or more markers of RA severity had an incidence rate of $7.47(95 \%$ CI $4.21-10.73)$ per 1,000 person-years.

\section{DISCUSSION}

Cardiovascular disease is a major comorbidity for patients with RA. However, relatively little information is available to determine which RA patients are at risk of $\mathrm{CV}$ events and the relative contributions of traditional CV risk factors versus markers of RA severity. We examined these issues using the CORRONA registry, a very large prospective RA cohort from the US. Both traditional CV risk factors and markers of RA severity contributed independent information to predict risk of future CV events. When both types of variables were included, the model's discrimination improved. Markers of RA severity added to the model's c-statistic above and beyond the traditional CV risk factors plus age and gender. Moreover, with increasing numbers of either type of variable, there was a trend toward increasing incidence rates of CV events.

Our data support the important independent contributions of both traditional CV risk factors and markers of RA severity to $\mathrm{CV}$ risk models. In models with both types of variables plus age and gender, we observed a c-statistic comparable to those calculated for analyses of the Framingham Risk Score. ${ }^{25}$ Our findings largely confirm prior work that focused on surrogate $\mathrm{CV}$ endpoints. ${ }^{17,18}$ While epidemiologic data do not substitute for treatment trials, these results suggest that strategies to reduce $\mathrm{CV}$ risk should focus on a strategy of controlling both traditional $\mathrm{CV}$ risk factors as well as controlling RA severity. In addition, it would be interesting to tailor interventions based on the risk factor profile. For example, patients with a greater number of markers of RA severity might be targeted for more intensive control of traditional $\mathrm{CV}$ risk factors, such as LDL lowering with statins, as well as better control of their RA disease activity.

There currently are no CV treatment trial data specific to RA patients. There are data from nonRA patients supporting the benefits of HMG CoA reductase inhibitors ("statins") for secondary prevention and primary prevention in high risk groups. ${ }^{26}$ In addition, the TARA trial found that statins effectively lower lipid levels in patients with RA and resulted in small decrements in CRP, while also modestly reducing disease activity.27 One large trial of statins for primary 
prevention of CVD events in patients with RA is ongoing, however no results are expected until at least 2011.28 Interestingly, a recently published trial of statin use in persons without hyperlipidemia but an elevated CRP found a significant reduction in CV events in the statin arm.29 The authors hypothesize that an independent anti-inflammatory effect of high-dose statin therapy could contribute to a diminished CV risk. If this is the case, it follows that other RA interventions which substantially reduce inflammation might also result in similar effects. Several observational studies found reduced CVD morbidity and mortality among methotrexate users. ${ }^{30,31}$ In addition, TNF antagonists may improve endothelial function. ${ }^{32}$ However, studies with actual CV endpoints demonstrate mixed findings. ${ }^{13}, 15,33$

The strengths of the current study are the large number of subjects with RA, the inclusion of traditional CV risk factors and markers of RA severity derived from both rheumatologists and patients, and the use of confirmed and adjudicated CV events. However, several limitations are important to discuss. First, while the CORRONA cohort is large, there were relatively few $\mathrm{CV}$ events included in this analysis, resulting in few events in certain cells in Table 4. The majority of endpoints included were confirmed by the treating rheumatologist, and the majority were adjudicated by a panel of cardiologists based on primary data. Medical records could not be obtained for some of the confirmed endpoints. These hospital medical records are not routinely received by U.S. rheumatologists when patients are hospitalized, and CORRONA does not have patient personal identifiers or patient consent to directly request these records. Thus, the investigator needs to obtain the records for CORRONA. Because some hospital records were requested for events occurring as remotely as 2002, records were not obtained on all patients. It would have been ideal to include coronary re-vascularization, but this information was not included in the CORRONA assessment forms used at the time these data were collected Second, it is possible that there is some under-ascertainment of CV events. Fewer composite events may have been observed because the data collection forms for the study period (2002-2006) did not have information on unstable angina. In addition, because death certificates were not available for the majority of patients, we chose to exclude CVrelated deaths for this study. However, based on reference populations such as the WHO MONICA Study, one might have anticipated 1.34 events per 1,000 person-years. ${ }^{34} \mathrm{We}$ found 3.98 events per 1,000 person-years in this cohort with RA, a group with a relative risk of $\mathrm{CV}$ events of $1.5-3.0$. Thus, we believe that under-ascertainment was not a major problem. It is also possible that the higher utilization rate for biologic DMARDs could have influenced the rate of $\mathrm{CV}$ events which we observed. Because of the large percentage of these patients who utilized biologic DMARDs or MTX, it is also possible that the incidence of CV events in this population would be lower than that described in European populations in which a much smaller percentage of patients with RA receive these agents.35 As has recently been described, diverse sources of epidemiologic information derived from different societies serve to enhance insights. 36 Third, detailed data on actual blood pressure and lipid measurements were not collected and thus it is possible that hypertension and hyperlipidemia were under-reported. Literature estimates of hypertension and hyperlipidemia in similar cohorts argues against under-reporting. 37,38 This limitation has been addressed in current versions of CORRONA forms. Finally, data on acute phase reactants are not mandated in CORRONA and therefore these data were incomplete. However, the Clinical Disease Activity Index (which does not require an acute phase reactant) is highly correlated with the Disease Activity Score (which does include an acute phase reactant). ${ }^{39}$

In conclusion, we found that both traditional CV risk factors and markers of RA severity were important predictors of future CV events in this very large US cohort. The risk added by RArelated factors added independent information to the predictive models. However, our models only explain a portion of the variability, likely because of imprecision in variable measurement as well as there being other variables not included in our adjusted models. These might include serologic markers, medications, and genetics. The European League Against Rheumatism has 
recently published recommendations for cardiovascular risk management among patients with RA. ${ }^{40}$ These recommendations acknowledge the importance of both traditional cardiovascular risk factors and RA-specific variables, similar to our analyses. The proportion of variation explained by both types of factors is similar, suggesting that both traditional CV risk factors and markers of RA disease severity can be appropriately targeted to lessen the incidence of $\mathrm{CV}$ events, the major source of mortality in patients with RA.

\section{Supplementary Material}

Refer to Web version on PubMed Central for supplementary material.

\section{Acknowledgments}

The Adjudication Committee included Drs. Michael Farkouh, Simonette Sawit and Sameer Bansilal.

Support: There was no specific support for these analyses. CORRONA has received general support in the last two years from Abbott, Amgen, BMS, Centocor, Genentech, Lilly, and Roche. Dr. Solomon receives salary support from research grants from the NIH (AR AR055989 and AR 047782), AHRQ, the Arthritis Foundation, Abbott, and Amgen. Jeffrey Curtis receives salary support from NIH (AR053351). Dr. Farkouh receives salary support from the NIH (HL071988 and HL092989). Dr Greenberg receives salary support from the NIH (K23AR054412) and the Arthritis Foundation.

\section{REFERENCES}

1. Gabriel SE. Cardiovascular morbidity and mortality in rheumatoid arthritis. Am J Med 2008 Oct;121 (10 Suppl 1):S9-S14. [PubMed: 18926169]

2. Chung CP, Oeser A, Raggi P, et al. Increased coronary-artery atherosclerosis in rheumatoid arthritis: relationship to disease duration and cardiovascular risk factors. Arthritis Rheum 2005 Oct;52:30453053. [PubMed: 16200609]

3. Solomon DH, Karlson EW, Rimm EB, et al. Cardiovascular morbidity and mortality in women diagnosed with rheumatoid arthritis. Circulation 2003;107:1303-1307. [see comment]. [PubMed: 12628952]

4. Wolfe F, Freundlich B, Straus WL. Increase in cardiovascular and cerebrovascular disease prevalence in rheumatoid arthritis. J Rheumatol 2003;30:36-40. [PubMed: 12508387]

5. Maradit-Kremers H, Crowson CS, Nicola PJ, et al. Increased unrecognized coronary heart disease and sudden deaths in rheumatoid arthritis: a population-based cohort study. Arthritis \& Rheumatism 2005;52:402-411. [PubMed: 15693010]

6. Goodson N, Marks J, Lunt M, Symmons D. Cardiovascular admissions and mortality in an inception cohort of patients with rheumatoid arthritis with onset in the 1980s and 1990s. Annals of the Rheumatic Diseases 2005;64:1595-1601. [PubMed: 15843450]

7. Solomon DH, Goodson NJ, Katz JN, et al. Patterns of cardiovascular risk in rheumatoid arthritis. Annals of the Rheumatic Diseases 2006;65:1608-1612. [PubMed: 16793844]

8. Dessein PH, Joffe BI, Stanwix AE. Inflammation, insulin resistance, and aberrant lipid metabolism as cardiovascular risk factors in rheumatoid arthritis. J Rheumatol 2003;30:1403-1405. [PubMed: 12858433]

9. Choi HK, Seeger JD. Lipid profiles among US elderly with untreated rheumatoid arthritis--the Third National Health and Nutrition Examination Survey. J Rheumatol 2005;32:2311-2316. [PubMed: 16331755]

10. van Halm VP, Peters MJ, Voskuyl AE, et al. Rheumatoid arthritis versus diabetes as a risk factor for cardiovascular disease, a cross sectional study. The CARRE Investigation. Ann Rheum Dis 2009;68:1395-1400. [PubMed: 18697775]

11. Del Rincon IWK, Stern MP, Freeman GL, O'Leary DH, Escalante A. Association between carotid atherosclerosis and markers of inflammation in rheumatoid arthritis patients and healthy subjects. Arthritis Rheum 2002;48:1833-1840. [PubMed: 12847676] 
12. Goodson NJ, Symmons DP, Scott DG, Bunn D, Lunt M, Silman AJ. Baseline levels of C-reactive protein and prediction of death from cardiovascular disease in patients with inflammatory polyarthritis: a ten-year followup study of a primary care-based inception cohort. Arthritis Rheum 2005;52:2293-2299. [PubMed: 16052597]

13. Dixon WG, Watson KD, Lunt M, Hyrich KL, Silman AJ, Symmons DP. Reduction in the incidence of myocardial infarction in patients with rheumatoid arthritis who respond to anti-tumor necrosis factor alpha therapy: results from the British Society for Rheumatology Biologics Register. Arthritis Rheum 2007;56:2905-2912. [PubMed: 17763428]

14. Goodson NJ, Wiles NJ, Lunt M, Barrett EM, Silman AJ, Symmons DP. Mortality in early inflammatory polyarthritis: cardiovascular mortality is increased in seropositive patients. Arthritis \& Rheumatism 2002;46:2010-2019. [see comment]. [PubMed: 12209502]

15. Solomon DH, Avorn J, Katz JN, et al. Immunosuppressive medications and hospitalization for cardiovascular events in patients with rheumatoid arthritis. Arthritis \& Rheumatism 2006;54:37903798. [PubMed: 17136752]

16. Wolfe F, Michaud K. The risk of myocardial infarction and pharmacologic and nonpharmacologic myocardial infarction predictors in rheumatoid arthritis: A cohort and nested case-control analysis. Arthritis Rheum 2008;58:2612-2621. [PubMed: 18759273]

17. Dessein PH, Norton GR, Woodiwiss AJ, Joffe BI, Wolfe F. Influence of nonclassical cardiovascular risk factors on the accuracy of predicting subclinical atherosclerosis in rheumatoid arthritis. $\mathrm{J}$ Rheumatol 2007;34:943-951. [PubMed: 17444592]

18. del Rincon I, Freeman GL, Haas RW, O'Leary DH, Escalante A. Relative contribution of cardiovascular risk factors and rheumatoid arthritis clinical manifestations to atherosclerosis. Arthritis Rheum 2005;52:3413-3423. [PubMed: 16255018]

19. Wilson PW, D'Agostino RB, Levy D, Belanger AM, Silbershatz H, Kannel WB. Prediction of coronary heart disease using risk factor categories. Circulation 1998;97:1837-1847. [PubMed: 9603539]

20. Collaborative overview of randomised trials of antiplatelet therapy--I: Prevention of death, myocardial infarction, and stroke by prolonged antiplatelet therapy in various categories of patients. Antiplatelet Trialists' Collaboration. BMJ 1994;308:81-106. [PubMed: 8298418]

21. Wolfe F. Which HAQ is best? A comparison of the HAQ, MHAQ and RA-HAQ, a difficult 8 item HAQ (DHAQ), and a rescored 20 item HAQ (HAQ20): analyses in 2,491 rheumatoid arthritis patients following leflunomide initiation. J Rheumatol 2001;28:982-989. [PubMed: 11361226]

22. Aletaha D, Smolen J. The Simplified Disease Activity Index (SDAI) and the Clinical Disease Activity Index (CDAI): a review of their usefulness and validity in rheumatoid arthritis. Clin Exp Rheumatol 2005;23(5 Suppl 39):S100-S108. [PubMed: 16273793]

23. Cook NR. Use and misuse of the receiver operating characteristic curve in risk prediction. Circulation 2007;115:928-935. [see comment]. [PubMed: 17309939]

24. Hurvich CM, Tsai CL. Model selection for extended quasi-likelihood models in small samples. Biometrics 1995;51:1077-1084. [PubMed: 7548692]

25. Kannel WB, McGee DL. Composite scoring--methods and predictive validity: insights from the Framingham Study. Health Services Research 1987;22:499-535. [PubMed: 3679841]

26. Third Report of the National Cholesterol Education Program (NCEP) Expert Panel on Detection, Evaluation, and Treatment of High Blood Cholesterol in Adults (Adult Treatment Panel III) final report. Circulation 2002;106:3143-3421. [PubMed: 12485966]

27. McCarey DW, McInnes IB, Madhok R, et al. Trial of Atorvastatin in Rheumatoid Arthritis (TARA): double-blind, randomised placebo-controlled trial. Lancet 2004;363:2015-2021. [PubMed: 15207950]

28. Service NH. TRACE-RA. Trial description. Available at: http://www.dgoh.nhs.uk/app/Default.aspx? alias=www.dgoh.nhs.uk/app/tracera.

29. Ridker PM, Danielson E, Fonseca FA, et al. Rosuvastatin to prevent vascular events in men and women with elevated C-reactive protein. N Engl J Med 2008;359:2195-2207. [PubMed: 18997196]

30. Choi HK, Hernan MA, Seeger JD, Robins JM, Wolfe F. Methotrexate and mortality in patients with rheumatoid arthritis: a prospective study. Lancet 2002;359:1173-1177. [see comment]. [PubMed: $11955534]$ 
31. van Halm VP, Nurmohamed MT, Twisk JW, Dijkmans BA, Voskuyl AE. Disease-modifying antirheumatic drugs are associated with a reduced risk for cardiovascular disease in patients with rheumatoid arthritis: a case control study. Arthritis Res Ther 2006;8:R151. [PubMed: 16984661]

32. Hurlimann D, Forster A, Noll G, et al. Anti-tumor necrosis factor-alpha treatment improves endothelial function in patients with rheumatoid arthritis. Circulation 2002;106:2184-2187. [PubMed: 12390945]

33. Solomon DH, Kremer JM, Curtis JR, et al. TNF Blocker Use and Cardiovascular Outcomes. Arthritis \& Rheumatism 2008;58:S1016.

34. Tunstall-Pedoe H, Kuulasmaa K, Mahonen M, Tolonen H, Ruokokoski E, Amouyel P. Contribution of trends in survival and coronary-event rates to changes in coronary heart disease mortality: 10-year results from 37 WHO MONICA project populations. Monitoring trends and determinants in cardiovascular disease. Lancet 1999;353:1547-1557. [PubMed: 10334252]

35. Kremer JM. The CORRONA database. Autoimmun Rev 2006;5:46-54. [PubMed: 16338211]

36. Zink A, Askling J, Dixon WG, Klareskog L, Silman AJ, Symmons DP. European biologicals registers: methodology, selected results and perspectives. Ann Rheum Dis 2009;68:1240-1246. [PubMed: 18647854]

37. Ong KL, Cheung BM, Man YB, Lau CP, Lam KS. Prevalence, awareness, treatment, and control of hypertension among United States adults 1999-2004. Hypertension 2007;49:69-75. [PubMed: 17159087]

38. Sempos CT, Cleeman JI, Carroll MD, et al. Prevalence of high blood cholesterol among US adults. An update based on guidelines from the second report of the National Cholesterol Education Program Adult Treatment Panel. JAMA 1993;269:3009-3014. [PubMed: 8501843]

39. Aletaha D, Nell VP, Stamm T, et al. Acute phase reactants add little to composite disease activity indices for rheumatoid arthritis: validation of a clinical activity score. Arthritis Res Ther 2005;7:R796-R806. [PubMed: 15987481]

40. Peters MJ, Symmons DP, McCarey D, et al. EULAR evidence-based recommendations for cardiovascular risk management in patients with rheumatoid arthritis and other forms of inflammatory arthritis. Ann Rheum Dis 2010;69:325-331. [PubMed: 19773290] 


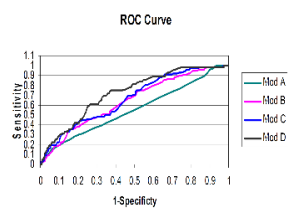

Figure 1.

The Figure illustrates the area under the receiver operating characteristic curves for four multivariable logistic models predicting cardiovascular events among subjects with rheumatoid arthritis: Model A contains only traditional cardiovascular risk factors (c statistic 0.57), Model B contains only markers of RA severity (c statistic 0.64 ), Model C contains both types of factors (c statistic 0.66) and Model D adds age and gender to Model C variables (c statistic 0.71). 
Table 1

Baseline characteristics of study population with rheumatoid arthritis in CORRONA ( $\mathrm{n}=10,156)$

\begin{tabular}{lc}
\hline & $\mathbf{N}(\%)$ or median (Inter-quartile range) \\
\hline Age, years, median & $59(50,69)$ \\
Female gender & $7,610(75.0 \%)$ \\
Duration of follow-up, months, median & $23(11,34)$ \\
Rheumatoid arthritis related variables & \\
RA duration, <24 months & $1,911(18.9 \%)$ \\
$\quad 24+$ months & $8,187(81.1 \%)$ \\
Seropositive & $4,852(72.2 \%)$ \\
Radiographic joint erosions & $3,445(50.7 \%)$ \\
Subcutaneous nodules & $2,019(20.1 \%)$ \\
Prior total joint arthroplasty & $3,067(30.4 \%)$ \\
Modified HAQ, < 1 & $8,707(87.8 \%)$ \\
$\quad 1-2$ & $1,124(11.3 \%)$ \\
$\quad 2+$ & $86(0.9 \%)$ \\
Clinical Disease Activity Index, median & $11(2,19)$ \\
Cardiovascular disease related variables & \\
History of MI & \\
History of cardiovascular disease & $376(3.7 \%)$ \\
History of diabetes & $859(8.5 \%)$ \\
History of hyperlipidemia drug use & $722(7.1 \%)$ \\
Fistory of hypertension & $937(9.2 \%)$ \\
\hline & $3,029(29.8 \%)$ \\
\hline & $1,601(16.1 \%)$ \\
\hline & $519(5.1 \%)$ \\
\hline
\end{tabular}

Notes: Abbreviations: RA, rheumatoid arthritis; IQR, interquartile range; HAQ, Health Assessment Questionnaire; MI, myocardial infarction.

*Seropositive refers to either rheumatoid factor or anti-CCP antibody. Radiographic joint erosions were based on the rheumatologist's report. History of CV disease includes a subject's report of myocardial infarction, stroke, transient ischemic attack, angina, or coronary intervention. Missing data exist for some variables: RA duration 58, serologic status 3549, radiographic erosions 3549, subcutaneous nodules 104, modified HAQ 81, and Clinical Disease Activity Index 772. 
Table 2

Cardiovascular event rates by age age and gender among CORRONA subjects with rheumatoid arthritis

\begin{tabular}{lccc}
\hline & Events $(\mathbf{n})$ & Patient-years & Event rate $\mathbf{( 9 5 \%}$ CI) \\
\hline Overall & 76 & 18,829 & $3.98(3.08-4.88)$ \\
Age $<75$ years & 51 & 15,975 & $3.19(2.32--4.07)$ \\
Age $\geq 75$ years & 25 & 2,651 & $7.92(4.55--11.30)$ \\
Female & 45 & 14,095 & $3.12(2.20--4.04)$ \\
Male & 31 & 4,724 & $6.56(4.26--8.87)$ \\
\hline
\end{tabular}

Notes: Cardiovascular events include myocardial infarction, stroke, and transient ischemic attack. Event rates are per 1,000 person-years. 


\section{Table 3}

Cardiovascular events among subjects in CORRONA with different numbers of traditional cardiovascular risk factors and markers of rheumatoid arthritis severity

\begin{tabular}{ccc}
\hline & $\mathbf{N}(\%)$ & \multicolumn{2}{c}{ Cardiovascular events } \\
Number of traditional CV risk factors & & 15 \\
0 & $3,463(34.1 \%$ & 26 \\
1 & $3,626(35.7 \%)$ & 35 \\
$2+$ & $3,067(30.2 \%)$ & \\
Number of markers of RA severity & & 3 \\
0 & $792(7.8 \%)$ & 16 \\
1 & $2,255(22.2 \%)$ & 16 \\
2 & $2,605(25.7 \%)$ & 41 \\
\hline $3+$ & $4,504(44.4 \%)$ & \\
\hline
\end{tabular}

Abbreviations: $\mathrm{CV}$, cardiovascular; RA, rheumatoid arthritis. Traditional CV risk factors include diabetes, hypertension, hyperlipidemia, tobacco use, known CV disease, a family history of premature CV disease, age $\geq 75$ years, and male gender. Markers of RA severity include disease duration $>5$ years, the presence of radiographic joint erosions, the presence of subcutaneous nodules, a prior total joint replacement, modified $\mathrm{HAQ}$ score $\geq 2$, a Clinical Disease Activity Index score > 22, and seropositivity. Percentages may not add to $100 \%$ due to rounding. 
Table 4

The incidence rate ( $95 \%$ confidence interval) of cardiovascular events by various combinations of traditional cardiovascular risk factors and markers of rheumatoid arthritis severity

\begin{tabular}{|c|c|c|c|c|}
\hline $\begin{array}{l}\text { RA factors } \\
\text { CV risk factors }\end{array}$ & 0 & 1 & 2 & $3+$ \\
\hline 0 & $\begin{array}{c}\mathrm{N}=305 \\
\text { Events }=0 \\
\text { Person-years }=502 \\
\text { Rate } 0(0-5.98)\end{array}$ & $\begin{array}{c}\mathrm{N}=820 \\
\text { Events }=5 \\
\text { Person-years }=1,512 \\
\text { Rate 3.31 }(0.41-6.20)\end{array}$ & $\begin{array}{c}\mathrm{N}=928 \\
\text { Events }=1 \\
\text { Person-years }=1,805 \\
\text { Rate } 0.55(0-1.64)\end{array}$ & $\begin{array}{c}\mathrm{N}=1,410 \\
\text { Events }=9 \\
\text { Person-years }=2,946 \\
\text { Rate } 3.06(1.06-5.05)\end{array}$ \\
\hline 1 & $\begin{array}{c}\mathrm{N}=287 \\
\text { Events }=2 \\
\text { Person-years }=469 \\
\text { Rate } 4.27(0-10.17)\end{array}$ & $\begin{array}{c}\mathrm{N}=803 \\
\text { Events }=7 \\
\text { Person-years }=1,422 \\
\text { Rate } 4.92(0.85-7.59)\end{array}$ & $\begin{array}{c}\mathrm{N}=923 \\
\text { Events }=5 \\
\text { Person-years }=1,684 \\
\text { Rate } 2.97(0.37-5.57)\end{array}$ & $\begin{array}{c}\mathrm{N}=1,613 \\
\text { Events }=12 \\
\text { Person-years }=3,315 \\
\text { Rate } 3.62(1.58-5.67)\end{array}$ \\
\hline $2+$ & $\begin{array}{c}\mathrm{N}=200 \\
\text { Events }=1 \\
\text { Person-years = 264 } \\
\text { Rate } 3.80(0-11.22)\end{array}$ & $\begin{array}{c}\mathrm{N}=632 \\
\text { Events }=4 \\
\text { Person-years }=1,013 \\
\text { Rate } 3.95(0.09-7.81)\end{array}$ & $\begin{array}{c}\mathrm{N}=754 \\
\text { Events }=10 \\
\text { Person-years }=1,222 \\
\text { Rate } 8.18(3.13-13.24)\end{array}$ & $\begin{array}{c}\mathrm{N}=1,481 \\
\text { Events }=20 \\
\text { Person-years }=2,678 \\
\text { Rate } 7.47(4.21-10.73)\end{array}$ \\
\hline
\end{tabular}

Incidence rate per 1,000 person-years (95\% confidence interval). Traditional CV risk factors include diabetes, hypertension, hyperlipidemia, tobacco use, known CV disease, a family history of premature CV disease, age $\geq 75$ years, and male gender. Markers of RA severity include disease duration $>5$ years, the presence of radiographic joint erosions, the presence of subcutaneous nodules, a prior total joint replacement, modified HAQ score $\geq$ 2, a Clinical Disease Activity Index score > 22, and seropositivity. 